\title{
Designing the dispersion for optimum supercontinuum bandwidth using picosecond pulses
}

Nikolov, Nikola Ivanov; Bang, Ole; Bjarklev, Anders Overgaard

Published in:

Optical Fiber Communications Conference, 2003. OFC 2003

Link to article, DOI:

10.1109/OFC.2003.1247457

Publication date:

2003

Document Version

Publisher's PDF, also known as Version of record

Link back to DTU Orbit

Citation (APA):

Nikolov, N. I., Bang, O., \& Bjarklev, A. O. (2003). Designing the dispersion for optimum supercontinuum bandwidth using picosecond pulses. In Optical Fiber Communications Conference, 2003. OFC 2003 IEEE. https://doi.org/10.1109/OFC.2003.1247457

\section{General rights}

Copyright and moral rights for the publications made accessible in the public portal are retained by the authors and/or other copyright owners and it is a condition of accessing publications that users recognise and abide by the legal requirements associated with these rights.

- Users may download and print one copy of any publication from the public portal for the purpose of private study or research.

- You may not further distribute the material or use it for any profit-making activity or commercial gain

- You may freely distribute the URL identifying the publication in the public portal 
change of the PSP (principal state of polarization), while simulation test may not consider these restrictions. The requirement on real time response means that the selected algorithm needs to be efficient to achieve fast (real time) response. The requirement on reliable response means that the selected algorithm needs to be globally convergent so that response is always in the correct direction wherever the starting point is. In this paper, we will consider the problem described in [1] and propose an algorithm to meet the challenges of these requirements.

\section{PULSE-WIDTH}

A very good introduction on PMD in optical fibers is provided in [2]. Results and formulae on optical pulse-width can be found in [1] [4] [5] [9] We list only the related results and formulae to form the optimization problem discussed in [1] (To avoid difficulty to express some mathematical formulae in word format and to save the space sometimes, we provide references but not details.) To solve this problem is equivalent to find the best polarization states of the input and the output to minimize the output pulse-width for the fiber transmission system described by transfer matrix Let $E(w)$ be the Fourier transform of the input pulse $\mathrm{E}(\mathrm{t})$. The time varying electric field at the input of an optical fiber is given by [4] as $\mathrm{E}_{1}=\mathrm{E}(w) \alpha$. The time varying electric field at the output of the optical fiber can be represented as $E_{0}=T(w) E(w) \alpha$, where $T(w)$ is Jones (transfer) matrix of the fiber. More specifically, the Jones matrix representing the pulse spreading in one section of bi-refringent optical fiber is given by [3] [5] $\mathrm{e}^{-\mathrm{jbw} \sigma / 2}$, where $w$ is the frequency, $b$ is the differential group delay (DGD) vector and $\sigma$ are the Pauli matrices. It is also suggested [1] [7] that the Jones matrix can be rewritten as $T(w)=T_{L}\left(w+w_{0}\right)$, where $w_{0}$ is the optical carrie frequency. Finally, it is possible (cf. [1]) to project all frequency components of $E_{0}$ onto a particular state of polarization. Let $\beta$ be the Jones vector of the output state of polarization. Therefore, the result of receiving $E_{0}$ on a state of polarization $\beta$ will be $E_{\beta}=\beta T_{L}(w) E(w) \alpha$, where * denotes complex conjugate. The purpose of this paper is to propose a new algorithm to select the polarization state vectors of $\alpha$ and $\beta$ such that the pulsewidth of $E_{\beta}$ is minimized. Let $\langle\vec{t}\rangle$ and $\left\langle\mathrm{t}^{2}\right\rangle$ be the first and second moments of the Gutput signal (see the definitions in [4]), the pulse-width is defined by $q(\alpha, \beta)=\left\langle t^{2}\right\rangle-\langle t\rangle^{2}$. The problem is reduced to Min $q(\alpha, \beta)$, s.t. $\|\alpha\|=\|\beta\|=1$.

3. THE NEW OPTIMIZATION ALGO-

\section{RITHM}

The problem described above is a typical nonlinear constrained optimization problem. In [1] Lagrange multiplier method was used for computer simulation to find the solution. Since Lagrange multiplier method is used for general nonlinear equality constrained optimization problem, it is not efficient for this very special problem. In addition, the set of complex nonlinear eigenvalue equations used in [1] are very difficul to be solved from the viewpoint of numerical analysis. Also, the convergence property of Lagrange multiplier method for this problem was not addressed in [1]. We will use optimization techniques on Riemannian manifold (cf. [11]) to solve the problem. This method searches optimiz ers along geodesics of the smooth surface prescribed by the constraint set, and therefore all iterations are feasible points.

Since $\alpha$ and $\beta$ are complex, we denote by $\alpha_{R}, \alpha$ $\beta_{R}$, and $\beta_{Y}$ the real and imaginary parts of $\alpha$ and $\beta$ respectively. Direct calculation will give the partial derivatives of $\mathrm{dq}(\alpha, \beta) / \mathrm{d} \alpha_{\mathrm{R}}, \mathrm{dq}(\alpha, \beta) / \mathrm{d} \alpha_{\mathrm{T}}$, $\mathrm{dq}(\alpha, \beta) / \mathrm{d} \beta_{\mathrm{R}}$, and $\mathrm{d} q(\alpha, \beta) / \mathrm{d} \beta_{\mathrm{I}}$ (we omit here the proofs to save the space, and omit the formulae of the derivatives because of the difficulty of expressing mathematical formulae by plain micro-soft word). It is also straightforward to verify that all these four derivatives are real valued The constraint can be rewritten as a product manifold of two spheres $\mathbf{M}=\mathbf{B S}_{1}{ }^{\mathrm{n}-1} \times \mathbf{B S}_{2}{ }^{\mathbf{n}-1}$, where $\mathbf{B S}_{1}^{n-1}=\left\{\left(\alpha_{R}, \alpha_{1}\right):\left\|\left(\alpha_{R}, \alpha_{1}\right)\right\|=1\right\}$, and $\mathbf{B S}_{2}{ }^{n-1}=\left\{\left(\beta_{R}, \beta_{I}\right):\left\|\left(\beta_{R}, \beta_{I}\right)\right\|=1\right\}$. The search for the minimizer will be carried out along the geodesic on the surface of $\mathbf{M}$ corresponding to the partial derivatives. For this purpose, we first find, based on the partial derivatives, the gradient of $q(\alpha, \beta)$, which is a projection of the derivative vector on to the tangent plan of $\mathbf{M}$ at the current position $(\alpha, \beta)$. Let $w_{1}=\left[\alpha_{R}, \alpha_{I}\right]^{\mathrm{T}}, w_{2}=\left[\beta_{R}, \beta_{I}\right]^{\mathrm{T}}$ Denote by diag( ) a diagonal matrix with its argument as the diagonal elements, and let $\mathrm{V}=\operatorname{diag}\left(\mathrm{w}_{\mathrm{j}}\right), \mathrm{i}=1,2$. The matrix that projects the derivative vector on to the tangent plan is then given by $(I-V V)$. The gradient vector of $q(\alpha, \beta)$ is therefore given by $\operatorname{grad}(q(\alpha, \beta))=\left(\mathrm{I}-\mathrm{VV}^{\mathrm{T}}\right)[\mathrm{dq}(\alpha, \beta)$ $\left.\mathrm{dw}_{1}, \mathrm{dq}(\alpha, \beta) / \mathrm{dw} \mathrm{w}_{2}\right]^{\mathrm{P}}=\left[\mathrm{h}_{1}, \mathrm{~h}_{2}\right]^{\mathrm{T}}=\mathrm{h}$. Let $\mathrm{H}_{\mathrm{i}}=\mathrm{h}_{\mathrm{i}} /\left\|\mathrm{h}_{\mathrm{i}}\right\|$ for $i=1,2$, if $h_{i} \neq 0$ or $H_{i}$ be any unit length vector otherwise. Denote $w=\left[w_{1}, w_{2}\right]^{T}, H=\left[H_{1}, H_{2}\right]^{T}$. Using the geodesic formula for sphere [8], it is easy to obtain the following

Claim: The geodesic of $\mathrm{M}$ emanating from $\mathrm{w}$ along the direction $h$ is given by $\exp _{w}(t h)=\operatorname{diag}\left(\cos \left(\| h_{i}|| t\right) I\right) w+\operatorname{diag}\left(\sin \left(\left\|h_{i}\right\| t\right) I\right) H$. From this claim, we can adopt a general algorithm proposed in [11] to the pulse-width compression problem.

Algorithm:

Data: Parameters $\lambda$ in $(0,1), \delta$ in $(0,1), \varepsilon>0$.

Step 0: Set $k=0$ and select $w^{0}$ on $M$. Then compute $\operatorname{grad}(q)\left(w^{k}\right)$

Step 1: Find $i_{k}=$ smallest $i$ such that $\varphi_{\mathrm{k}}=\mathrm{q}\left(\operatorname{diag}\left(\cos \left(\left\|\mathrm{h}_{\mathrm{i}}\right\| \delta^{\mathrm{i}}\right) \mathrm{I}\right) \mathrm{w}+\operatorname{diag}\left(\sin \left(\left\|\mathbf{h}_{\mathrm{i}}\right\| \delta^{\mathrm{i}}\right) \mathrm{I}\right) \mathrm{H}\right)$

$-q\left(w^{k}\right) \geq \lambda \delta^{i}\left\|\operatorname{grad}(q)\left(w^{k}\right)\right\|^{2}$ holds.

Step 2: Update $w^{k+1}=\operatorname{diag}\left(\cos \left(|| h_{i} \| \delta^{j}\right) I\right) w$

+ diag $\left(\sin \left(\left\|h_{\mathrm{i}}\right\| \delta^{\mathrm{i}}\right) \mathrm{I}\right) \mathrm{H}$.

Step 3: Compute $\operatorname{grad}(q)\left(w^{k+1}\right)$.

If $\left\|\operatorname{grad}(q)\left(w^{k+1}\right)\right\| \leq \varepsilon$, stop; otherwise increase $k$ by one then go back to Stepl.

It is clear that the iteration in step 1 , which involves the calculation of the derivatives and projection, is much more straightforward and simpler than the iteration described in [1], which involves to solve a set of complex nonlinear eigenvalue equations. Several properties were proved in [11] about the algorithm. First, inequality in step 1 is well defined in the sense that one can always find an i such that the inequality holds. This means the search will never fail. Second, the objective function is improved in every iteration, and all intermediate points obtained in Step 2 are feasible, i.e., they meet the constraint. This feature is important for real time applications. Third the algorithm is globally convergent to a set of optimizer candidates. Finally, the convergence rate is linear.

This proposed algorithm is implemented in MAT. $\mathrm{LAB}$ for a simple problem proposed in [1]. Starting from any initial guess, after a few iterations, the algorithm finds a local optimizer.

\section{CONCLUSIONS}

A new algorithm is proposed to optimize a pulsewidth compression problem. This algorithm is better for real time implementation. Since many PMD compensation problems are constrained optimization problem with variables as Jones vectors, this technology can easily be extended to all those cases.

\section{REFERENCE}

[1] L. Chen and M. Yanez, C. Huang and X. Bao, Journal of Lightwave Technology, Vol. 19, pp. $830.836,2001$

[2] J. P. Gordon and H. Kogelnik, PNAS Vol. 97, pp 4541-4550, 2000.

[3] B. Hutter and N. Gisin, Optics letters, Vol 22, pp. 504-506, 1997

[4] M. Karlsson, Optics Letters, Vol. 23, pp. 688690, 1998.

[5] P. Lu and L. Chen and X. Bao, Journal of Lightwave Technology, Vol. 19, pp. 856-860, 2001.

[6] E. Polak, Computational Methods in Optimization, Academic Press, New York, 1971.

[7] J. G Proakis, Digital Communications, Third Edtion, McGraw Hill, New York, 1995.

[8] J. G. Ratcliffe, Foundations of Hyperbolic manifolds, Springer-Verlag, New York, 1994.

[9] W. Shieh, IEEE Photon. Technol. Letter, Vol. 11 , pp. $677-679,1999$.

[10] S. Shin and I. Yeo and H. Song, Proceedings of Optical Fiber Conference, LA, USA, 2001.

[11] Y, Yang, Proceedings of the 37 th Control and Decision Conference, USA, 1998.
MF16 5:30 PM

Designing the Dispersion for Optimum Supercontinuum Bandwidth Using Picosecond Pulses

N. I. Nikolov, Tech. University of Denmark, Kgs. Lyngby, Denmark; N. I. Nikolov, Riso Natl. Lab., Roskilde, Denmark; O. Bang, Tech. University of Denmark, Kgs. Lynby, Denmark; A. Bjarklev, COM, Tech. University of Denmark, Kgs. Lyngby, Denmark,Email: nin@imm.dtu.dk.

Generation of a $800 \mathrm{~nm}$ wide within $20 \mathrm{~dB}$ supercontinuum with picosecond pulses is possible by proper design of the dispersion to enhance the efficiency of the direct degenerate four-wavemixing. The robustness to random fluctuations along the fiber is also improved.

Introduction.

Due to their unusual dispersion properties and high effective nonlinearities, photonic crystal fibers (PCFs) [1] and tapered fibers [2] are promising sources for efficient supercontinuum generation (SCG). The dispersion and nonlinearity characteristics of these fibers and the advantage that their dispersion may be modified by a proper design of the cladding structure [3], and by changing the degree of tapering [2], respectively, makes them a convenient tool for SCG. Using femtosecond pulses a supercontinuum (SC) spanning 400$1500 \mathrm{~nm}$ has been generated in a PCF [1], and a tapered fiber [2]. The wide SC was later explained to be a result of self-phase modulation (SPM) and direct degenerate four-wave-mixing (FWM) [4]. It has been shown, that complex high-power femtosecond lasers are not necessary, - SCG may be achieved with picosecond and even nanosecond pulses. Thus Coen et al. obtained a SC in a PCF using sub-kilowatt picosecond pulses and showed that the primary mechanism was the combined effect of stimulated Raman scattering (SRS) and parametric FWM, allowing the Raman shifted components to interact efficiently with the pump $[5,6]$. Here we show that the use of direct degenerate FWM may significantly improve both the efficiency and robustness of SCG with sub-kilowatt picosecond pulses in PCFs, if the dispersion is properly designed.

Effective SCG with picosecond pulses in PCFs using degenerate FWM.

Here we optimise the dispersion profile and study the SCG process numerically using the well known coupled nonlinear Schroedinger equations that describe the evolution of the $\mathrm{x}$ - and y-polarization components of the field for pulses with a spectral width up to $1 / 3$ of the pump frequency $[5,6]$. This model accounts for SPM, cross-phase-modulation, FWM, and SRS. An initial random phase noise seeding of one photon per mode is included.

We consider the same PCF and numerical and experimental data as in $[5,6]$. We pump along the slow axis with $30 \mathrm{ps}$ pulses of $\mathrm{I}_{\mathrm{p}}=400 \mathrm{~W}$ peak power and pump wavelength $\lambda_{p}=647 \mathrm{~nm}$. Our PCF has dispersion $D\left(\lambda_{\mathrm{p}}\right)=-30 \mathrm{ps} /(\mathrm{nm} \cdot \mathrm{km})$, zero dispersion wavelength $\lambda_{2}=675 \mathrm{~nm}, \mathrm{n}_{2}=3 \times 10^{-20} \mathrm{~m}^{2} / \mathrm{W}$, and birefringence $\mathrm{n}_{\mathrm{x}}-\mathrm{n}_{\mathrm{y}}=1.9 \times 10^{-6}$. The dispersion is expanded around the pump to include $\beta_{2}=7.0 \mathrm{ps} 2$ $\mathrm{km}, \quad \beta_{3}=5,1 \times 10^{-2} \mathrm{ps}^{3} / \mathrm{km}, \quad \beta_{4}=-4.9 \times 10^{-5} \mathrm{ps}^{4} / \mathrm{km}$, $\beta_{5}=1.2 \times 10^{-7} \mathrm{ps}^{5} / \mathrm{km}, \beta_{6}=-1.8 \times 10^{-10} \mathrm{ps}^{6} / \mathrm{km}$, and $\beta_{7}=1.2 \times 10^{-13} \mathrm{ps}^{7} / \mathrm{km}$. A uniform loss of $0.1 \mathrm{~dB} / \mathrm{m}$ is used and the effective area is approximated with the core area $\mathrm{A}_{\text {core }}=1.94 \mu \mathrm{m}^{2}$, giving the nonlinearity parameter $\gamma=2 \pi n_{2} /\left(\lambda_{\mathrm{p}} \mathrm{A}_{\text {core }}\right)=0.15(\mathrm{Wm})^{-1}$ 

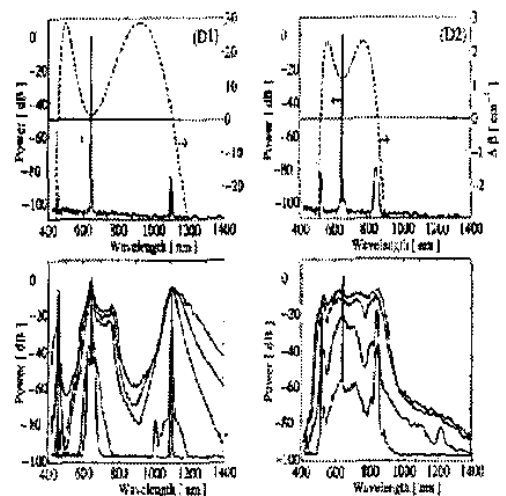

.Fig.1 Upper row: phase-mismatch $\Delta \beta$ and spectrum of the slow axis polarization component at $\mathrm{L}=10 \mathrm{~cm}$. Bottom row: same spectrum at $\mathrm{L}=20 \mathrm{~cm}$, $30 \mathrm{~cm}$, and $2 \mathrm{~m}$. Left figures are for dispersion profile D1 and rigth figures for D2, respectively. For case D1 additional spectral line is shown for $\mathrm{L}=3.7 \mathrm{~m}$

We use the standard split-step Fourier method with $2^{17}$ points in a time window of $T=236 \mathrm{ps}$. In with 2 points in a time window of $T=236 \mathrm{ps}$. In
our longest simulation out to $L=3.7 \mathrm{~m}$ the photon number is conserved to within $5 \%$ of its initial value. Due to our large spectral window $(405 \mathrm{~nm}>1613 \mathrm{~nm})$ we see in Fig.1(left) the emergence of FWM stokes and anti-stokes waves at the wavelengths $\lambda_{\mathrm{s}}=1100 \mathrm{~nm}$ and $\lambda_{\mathrm{as}}=458 \mathrm{~nm}$ for which the phase matching condition $\Delta \beta=\beta_{\mathrm{s}}+\beta_{\mathrm{as}}$ $2 \beta_{p}+\gamma I_{p}=0$ is satisfied. The spectral window presented in [6] was narrower and thus $\lambda_{\mathrm{s}}$ and $\lambda_{\text {ss }}$ were not observed. We find the maximum FWM parametric gain to be twice the maximum SRS gain, which explains why the FWM stokes and anti-stokes components appear before the SRS components.

The loss and walk-off of the PCF gives the maximum distance $\mathrm{L}_{\mathrm{max}}$ over which nonlinear processes, and thus the SCG process, are efficient. From Fig.1 we see that after the FWM stokes and anti-stokes components are generated they broaden much in the same way as the central part of the spectrum around the pump. The merging of the spectral parts around $\lambda_{a s}, \lambda_{p}$, and $\lambda_{s}$ would create an ultra broad spectrum as observed in tapered fibers with femtosecond pulses $[2,4]$. However, in this particular case the FWM stokes and antistokes lines are too far away for a merging to take place within the maximum length $L_{\max }$, i.e., before nonlinear effects become negligible.

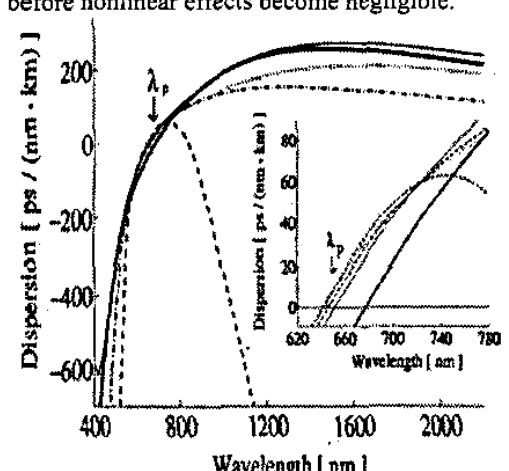

Fig.2 Original dispersion [5] D1 (thick solid line) and our modified dispersion D2 (thin solid line) D3 (dotted line), D4 (dash-dotted line), and DS (dashed line).

The wavelengths $\lambda_{\mathrm{s}}$ and $\lambda_{\mathrm{as}}$ can be adjusted to be closer to the pump wavelength $\lambda_{\text {by a proper }}$ design of the dispersion. This will enable the FWM stokes and anti-stokes lines to broaden enough to allow a final merging. To show the effect we modify $\beta_{2}, \beta_{4}$, and $\beta_{6}$ to $\beta_{2}=1.0 \mathrm{ps}^{2} / \mathrm{km}$, $\beta_{4}=-2.5 \times 10^{-5} \mathrm{ps}^{4} / \mathrm{km}_{2}$, and $\beta_{6}=-3.25 \times 10^{-10} \mathrm{ps}^{6} / \mathrm{km}$.

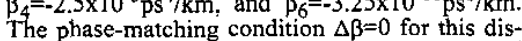
persion profile $D 2$ then gives $\lambda_{s}=850 \mathrm{~nm}$ and $\lambda_{\mathrm{as}}=530 \mathrm{~nm}$

The numerical results shown in Fig.1 confirm our hypothesis. The FWM stokes and anti-stokes lines are still widely separated, but now generated close enough to the pump to broaden and merge. The resulting ultrabroad $\mathrm{SC}$ is flat within $20 \mathrm{~dB}$ and spans $510 \mathrm{~nm}$ in contrast to the original $230 \mathrm{~nm}$ observed in [6].

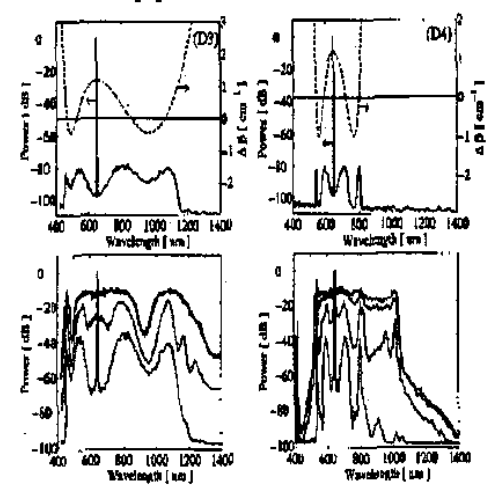

Fig.3 Upper row: phase-mismatch $\Delta \beta$ and spectrum of the slow axis polarization component at $\mathrm{L}=10 \mathrm{~cm}$. Bottom row: same spectrum at $\mathrm{L}=20 \mathrm{~cm}$, $30 \mathrm{~cm}, 1 \mathrm{~m}$, and $2 \mathrm{~m}$. Left: dispersion profile $\mathrm{D} 3$. Rigth: dispersion profile D4.
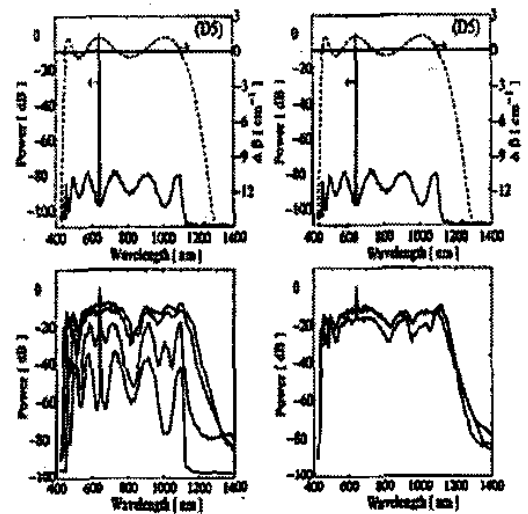

Fig.4 Upper row: phase-mismatch $\Delta \beta$ and spectrum of the slow axis polarization component a $\mathrm{L}=10 \mathrm{~cm}$. Bottom row: same spectrum at $\mathrm{L}=20 \mathrm{~cm}$ $30 \mathrm{~cm}, \mathrm{~lm}$, and $2 \mathrm{~m}$ for an input power of $400 \mathrm{~W}$ (left figure) and $500 \mathrm{~W}$ (right figure), at $\mathrm{L}=40 \mathrm{~cm}$, $1 \mathrm{~m}$, and $2 \mathrm{~m}$.

In Fig. 3, we show the spectrum for the dispersion profiles D3-D4, which have two Stokes and two anti-Stokes lines. The corresponding dispersion coefficients $\beta_{2}, \beta_{4}$, and $\beta_{6}$ are $\beta_{2}=-0.28 \mathrm{ps}^{2} / \mathrm{km}$, $\beta_{4}=0.05 \times 10^{-5} \mathrm{ps}^{4} / \mathrm{km}$, and $\beta_{6}=-0.29 \times 10^{-10} \mathrm{ps}^{6} / \mathrm{km}$ for $\mathrm{D} 3$, and $\beta_{2}=-1.3 \mathrm{ps}^{2} / \mathrm{km}, \beta_{4}=-2.6 \times 10^{-5} \mathrm{ps}^{4} / \mathrm{km}$, and $\beta_{6}=-58.8 \times 10^{-10} \mathrm{ps}^{6} / \mathrm{km}$ for $\mathrm{D} 4$. For the dispersion profile $\mathrm{D} 4$, cascaded direct degenerate FWM peaks appear. This leads to an even broader SC of approximately $600 \mathrm{~nm}$ within $20 \mathrm{~dB}$.

For the dispersion profile D5 we have $\beta_{2}=-1.1 \mathrm{ps}^{2} /$ $\mathrm{km}, \beta_{4}=2.74 \times 10^{-5} \mathrm{ps}^{4} / \mathrm{km}$, and $\beta_{6}=-3.92 \times 10^{-10} \mathrm{ps}^{6} /$ $\mathrm{km}$. In Fig. 4 the spectrum for the dispersion profile D5 is shown and for this case the direct degenerate FWM process generates three Stokes and anti-Stokes lines. As seen this improves a lot the spectrum for shorter fiber lengths. For longer lengths, as $\Delta \beta=\Delta \beta\left(I_{p}\right)$, the wavelengths of the Stokes and anti-Stokes lines shift due to the input power depletion and dips in the final SC spectrum are formed. To overcome this feature we increase slightly the input power to $I_{p}=500 \mathrm{~W}$, and as is seen from Fig. 4 the resulted spectrum is $800 \mathrm{~nm}$ wide within $20 \mathrm{~dB}$.
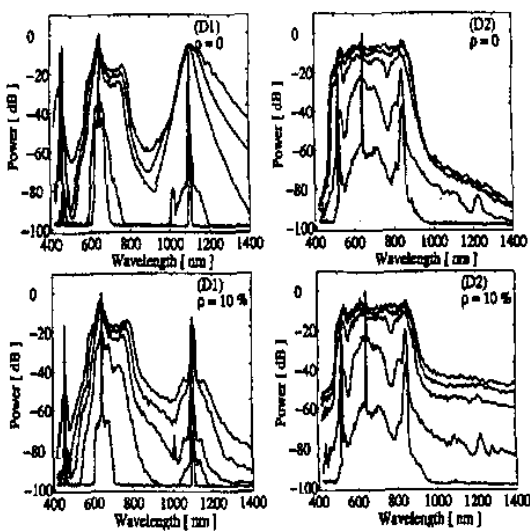

Fig. 5 Spectrum of the slow axis polarisation component at $\mathrm{L}=20 \mathrm{~cm}, 30 \mathrm{~cm}, 1 \mathrm{~m}, 2 \mathrm{~m}$, and $3.7 \mathrm{~m}$. Upper row: without noise. Bottorn row: with noise corresponding to $10 \%$ variation. Left: dispersion profile D1. Right: dispersion profile D2.

The absence of Stokes and anti-Stokes lines from the direct degenerate FWM process in the experiments in [6], is explained with the violation of the phase matching condition $\Delta \beta$ due to irregularities along the fiber. We estimate the robustness of the proposed method for SCG, towards random dispersion fluctuations along the fiber. We consider the highly nonlinear PCF fiber reported in [7], and estimate the variation of the dispersion coefficients $\beta_{2}$ and $\beta_{3}$ to be: $\left\langle\beta_{2}>\cong<\beta_{3}>=9.5 \%\right.$. We assume that the variation $\left\langle\beta_{k}\right\rangle$ for all dispersion coefficients $\beta_{2-7}$ is $10 \%$. The random process is Gaussian white noise. From Fig.5 it is seen that for dispersion profile D1, the random dispersion variations significantly reduce the effectiveness of the direct degenerate FWM and it finally does no contribute to the generated SC. This is explained by the narrow gain-band for direct degenerate FWM for the dispersion profile Dl, strongly reducing the average gain. However for dispersion profile D2, the generation of the Stokes and anti-Stokes lines is more robust, due to the broader gain band-width. By a more thorough theoretical study it can be shown that for dispersion profiles D3-D5 the band-width of the direct degenerate FWM gain profile is even broader This allows us to conclude that for the modified dispersion profiles D2-D5, the improvement of SCG due to generation and merging of Stokes and anti-Stokes lines is robust to fiber imperfections. Conclusion.

We have shown that the direct degenerate FWM can significantly improve the SCG due to broadening and final merging of the generated stokes and anti-stokes lines. The bandwidth of the achieved SC can be optimized by a proper design of the dispersion. The robustness of the process towards variations in the dispersion coefficients along the PCF was also investigated. It is shown that for a fiber with a modified dispersion profile the generation and final merging of the Stokes and anti-Stokes lines with the main SC part is robust. This work was supported by the Danish Technical Research Council (Grant no. 26-00-0355) and the Graduate School in Nonlinear Science (The Danish Research Agency).

References

1. J. K. Ranka et. al., Opt. Lett., 25 (2000), p. 25.

2. T. A. Birks et. al., Opt. Lett., 25 (2000), p. 1415

3. A. Ferrando et. al., Opt. Lett., 25 (2000), p. 7902.

4. A. V. Gusakov et. al., QELS, (2001), p. 29.

5. S. Coen et. al., Opt. Soc. Am. B, 19 (2002), p. 753.

6. N. I. Nikolov, et. al., ECOC 2002, P1.36.

7. K. P. Hansen et. al., OFCl02 Post Deadline FA 9 (2002). 